\title{
La importancia de rastrear el pensamiento de Aristóteles en la Nueva España
}

Virginia Aspe Armella

Universidad Panamericana, Campus Ciudad de México, México

Recibido el 18/12/2019/Aceptado el 28/02/2020

\begin{abstract}
Resumen
Este escrito da razones externas e internas por las que es necesario acuñar el término "aristotelismo novohispano". La autora de cuenta de la necesidad de pensar la filosofía desde la lengua vernácula y conociendo las tradiciones filosóficas que formaron el horizonte de comprensión del pensamiento iberoamericano. En la primera parte del texto, la autora expone la génesis del aristotelismo salmantino del siglo XV, la formación académica de los frailes venidos a América en el siglo XVI y analiza el caso concreto de Nueva España en la cátedra y polémicas. La segunda parte del texto presenta argumentaciones formuladas por autores de la época.
\end{abstract}

\section{The importance of tracking Aristotle's thought in New Spain}

\begin{abstract}
This text provides external and internal reasons for which it is necessary to coin the term "Aristotelianism of New Spain". The author explains the need for thinking philosophy from the perspective of one's vernacular language and for knowing the philosophical tradition that formed Ibero-American thought's horizon of understanding. In the first part of the text, the author explains the origin of Salamancan Aristotelianism in the 15th century and the academic education of the friars that came to America in the $16^{\text {th }}$ century, and she analyses the specific case of New Spain's lectures and polemics. The second part of the text provides arguments made by authors of that period.
\end{abstract}

\section{Introducción}

En esta investigación expongo las razones de la importancia del análisis de la tradición aristotélica iberoamericana poniendo un caso de protagonismo aristotélico: el período novohispano en México. Pese a que este aristotelismo no tuvo la raigambre de otras tradiciones aristotélicas (como la tradición aristotélico-neoplatónica, la aristotélico-tomista, la aviceniana, la averroísta en sus múltiples asimilaciones, la

\section{Palabras clave}

Nueva España aristotelismo salmantino tradiciones filosóficas humanismo renacentista nominalismo

\section{Keywords}

New Spain

Salamancan Aristotelianism philosophical traditions Renaissance humanism nominalism 
peripatético-renacentista, etc.), hay razones para seguir la tradición iberoamericana del aristotelismo: la primera razón, que es externa a la filosofía, estriba en la necesidad de pensar a partir de la lengua y la geografía; la segunda, que es interna, se debe a la impronta cultural a partir de la cual se desarrollan las ideas aristotélicas en América, con un replanteamiento de sus tesis filosóficas a partir de la experiencia del otro americano, la necesidad de clasificar la realidad recién descubierta en el entorno natural, los climas y su geografía, y el impacto ante las nuevas prácticas autóctonas.

A pesar de que el idioma español ha sido incluido ya en los congresos mundiales de filosofía como una de sus lenguas universales, los especialistas en filosofía antigua desconocen en muchas ocasiones la tradición filosófica aristotélica y la de otras filosofías clásicas de América; dicho aprendizaje sería valioso para precisar los términos utilizados por la propia lengua: en el ámbito filosófico es de primer orden el conocimiento de la evolución de los términos. No se quiere decir con esto que la filosofía se ate a la historia y la tradición; tampoco que el estudioso de la filosofía antigua haya de ocuparse de la tradición filosófica local, sino que el filósofo ha de tener conciencia de dónde está inserto: de conocer desde dónde piensa y cómo mira los problemas. Ya que la filosofía occidental y griega son más acción vital que historia, más un pensar los problemas de la realidad en diálogo y comunidad que decir, técnicamente y con precisión, qué dijeron otros, importa tener al menos un mínimo conocimiento de las tradiciones filosóficas de donde se parte: saber, al menos, cómo surgieron y qué vigencia tienen sus argumentaciones.

La tradición antigua de la filosofía iberoamericana enfrenta problemas específicos por su lengua; los especialistas señalan que el español fue la última lengua que se incorporó al cambio del latín (no ocurrió sino hasta con Feijoo en 1726, cuando Bacon, Descartes y Vico lo habían logrado en su nación un siglo antes). El punto es clave si entendemos la filosofía como diálogo y cultura.

Otro obstáculo que enfrenta la filosofía iberoamericana es que su manera de reflexionar sobre los principios de la realidad fue a través del ensayo; respecto a esto, la opción analítica en América ha propiciado un desarrollo de la filosofía con significaciones asépticas más precisas. Por la peculiaridad de su origen (la filosofía antigua en Iberoamérica surge de tradiciones opuestas al poder del coloniaje), esta filosofía debe cuidar especialmente sus exigencias metódicas; pero la particularidad del origen filosófico americano tiene también beneficios: involucrar la impronta clásica del humanismo para explicar la realidad del entonces llamado neófito o gentil, la conciencia de una cultura híbrida originaria y la herencia de una filosofía neo-medieval fuerte en categorías lógicas que la hizo apta para la universalización. ${ }^{1} \mathrm{El}$ punto nos lleva a repensar nuestra tradición filosófica iberoamericana sin tener por ello que estar insertos en esa línea de investigación.

Presentaré aquí el caso del aristotelismo novohispano para probar mi propuesta.

El aristotelismo novohispano cuenta con elementos externos e internos en su formulación que lo hacen merecedor de una mayor consideración por parte del público filosófico. La manera en que probaré esta hipótesis es dando las razones externas o de contexto, e internas o de argumentación, que lo comprueban. En la primera parte de mi escrito daré cuenta, de manera general, de las razones externas que justifican mi aproximación, a saber: a) el contexto de las tradiciones aristotélicas venidas de la Universidad de Salamanca y la Escuela formada por ella, b) otras tradiciones aristotélicas que llegaron a la Nueva España en la época, y c) por el currículo de la Real y Pontificia Universidad de México. En una segunda parte del escrito daré cuenta de las razones internas por las que es importante la tradición aristotélica novohispana mediante cuatro puntos: a) la experiencia del otro en América y el impacto filosófico 
que tuvo Aristóteles en la solución de la problemática, b) los argumentos aristotélicos cara a la organización política de la Nueva España, c) la asimilación de la tradición aristotélico-naturalista al clasificar la realidad física del nuevo orbe, y d) una novedosa propuesta de acceso al Organon y al De anima de Aristóteles frente a las escuelas aristotélicas de su tiempo.

\section{Las cuestiones de contexto que justifican el protagonismo de Aristóteles en la Nueva España}

Una cuestión de contexto que debe rastrearse es el antecedente novohispano del aristotelismo ibérico. Como se sabe, la fundación de la Real y Pontificia Universidad de México en 1553 coincide en estructura con las universidades medievales, en especial con la Universidad de Salamanca, que fue fundada en $1218 \mathrm{y}$, posteriormente, con las normativas de Alfonso X, que fija el currículo con la prioridad de la teología sobre las facultades de Artes, Derecho y Medicina. Ya desde 1271 Salamanca cuenta con aristotélicos como Gil Zamora (1241-1318; cfr. su obra Historia naturalis), que traduce textos de Aristóteles del árabe al latín, y Pedro Gallego Cartagena, quien en 1267 traduce las obras de Aristóteles del árabe al latín (cfr. Summa Astronomica, MS. 8919 de Manuel Castro, Biblioteca Nacional de Madrid). ${ }^{2}$ Lo que se hacía entonces era transcribir el texto de Aristóteles poniéndolo en dos lenguas, el latín y el árabe; se incluían adiciones pedagógicas, estilísticas y terminológicas, y se realizaba una rearticulación del texto original en función de un esquema distinto.

Para el siglo XV, la Universidad de Salamanca introduce en su currículo la exposición de la Ética a Nicómaco de Aristóteles; antes, el currículo destacaba las obras naturales, el Órganon y la Metafísica. El vuelco será crucial en el destino americano debido a que los egresados de la Universidad de Salamanca que fueron enviados a América por motivos evangelizadores se habrán formado en la impronta salmantina de la denominada "primera etapa" de la Escuela de Salamanca (para distinguirla de la "segunda", liderada por Francisco de Vitoria, Domingo Soto y Domingo Báñez; con este último concluye la segunda etapa de la Escuela de Salamanca y comienza la denominada Escolástica barroca de Suárez y los jesuitas en Portugal).

Lo que pasó en el siglo XV en la Universidad de Salamanca es relevante para nuestro estudio y ha sido soslayado por los aristotélicos actuales debido al protagonismo que se ha dado a Francisco de Vitoria, una relevancia tan grande en la Escuela de Salamanca que casi se identifica su pensamiento escolástico con la Escuela. Pero está documentado que en el siglo XV hay tres cátedras aristotélicas en el Colegio Viejo de San Bartolomé: la de Alonso de Madrigal -"el Tostado"-,la de su discípulo Pedro Osma y la de Fernando Roa. ¿Por qué y cómo entró a Salamanca este peculiar aristotelismo? (Cfr. Castillo Vegas, 2004; Aspe Armella, 2014, 2018; y Sabido Sánchez-Juárez, 2016).

Durante las cátedras de Osma y Roa en la facultad de Artes de Salamanca surge la propuesta de formar alumnos que realicen un proyecto republicano español. En el siglo XV se forma una burguesía que comienza a ocupar los puestos medios del gobierno y la corona; los miembros de esta burguesía, egresados de la facultad de Artes de Salamanca, ocuparán cargos en la administración gubernamental, no seguirán los estudios en la facultad de teología ni serán siempre clérigos; se interesan por el bien común de España promoviendo equilibrios entre la monarquía y las cortes amparados en el estudio de la Ética y la Política de Aristóteles. Está documentado que fueron estos estudiantes con intereses en la filosofía práctica los que pidieron a sus maestros que incluyeran las obras prácticas de la filosofía de Aristóteles. El proyecto promovía los intereses de las cortes y de la aristocracia; fue así como se comenzó a realizar la transcripción de estas obras que autores previos, como Pedro
2. Se trata del conocido enciclopedismo español. Cfr. Martínez Gázquez (1995), García Ballester (1994) y Coll (1944). 
3. Para este tema, cfr. las investigaciones de José Alonso Labajos, Cirilo Flores Miguel y otros especialistas que analizan los comentarios a la Ética de Aristóteles en las facultades de Derecho y Artes.
Gallego (1244), denominado "el Santo Alfaquí", preso en Cartagena en su expedición en Murcia, y Pedro Díaz de Toledo (1379), "Gran Maestre de Rodas", apresado por los turcos en 1379, conocieron el pensamiento de Aristóteles por los árabes y lo dieron a conocer en Hispania, retomándose estos textos en el siglo XV.

Para este siglo tenemos dos corrientes aristotélicas en Salamanca por el intercambio entre oriente y occidente (Cfr. Herrero Prado (1998); Round (1966)). Las corrientes se desarrollaron en Aviñón con la tradición hispanojudía-musulmana, que culmina con Averroes, y la escolástico-tomista de Nápoles y Sicilia, que culmina con Suárez; ambas conformaron las tradiciones aristotélicas que, aunadas a la nueva estructura de saberes de la Universidad de Salamanca (impactada en el siglo XV por la Constitución de Martín $V$, que propiciaba la intensificación de la filosofía práctica), incidieron en la administración del gobierno, la monarquía, los patronazgos y el clientelismo emergente. Es así que la Primera Escuela de Salamanca (1406-1516) inserta las obras prácticas de Aristóteles. ${ }^{3}$

Si seguimos el impacto que estas lecturas tuvieron, podemos relacionar la impronta aristotélica con el movimiento comunero español del siglo XVI en el que se levanta Castilla, teniendo a Toledo como principal representante en la guerra de las comunidades (1519-1522); éstas, pese a que se rinden al final, hacen que Carlos I de España y V de Alemania haga concesiones en los equilibrios de poder con las cortes. Aragón se había unido a las protestas y queda en la impronta española un conflicto entre las ciudades, la burguesía emergente y la nobleza.

La aparición del aristotelismo político español del siglo XV es relevante. Este estudio de las cátedras aristotélicas desmitifica la frecuente interpretación de que el aristotelismo español es distinto del italiano y que, antes de la llegada del humanismo italiano en el siglo XVI, no había un aristotelismo español de raigambre salvo por pensadores árabes como Averroes o por el tomismo de Vitoria. El hecho desmitifica también la frecuente interpretación de que el humanismo italiano entró a Salamanca hasta que se formaron los estatutos de 1529 con Pérez de Oliva. Autores como A. R. D. Pagden en "The Diffusion of Aristotle's Moral Philosophy in Spain, ca. 1400 - ca. 1600" (1975), que estudiaron manuscritos catalanes, valencianos, gallegos y portugueses en la Biblioteca del Escorial, y otros como Charles Lohr, sostuvieron que Aristóteles sólo apareció en la Biblioteca Tesoro de Bruneto Latini en versión castellana después de 1400. Ellos separaron tajantemente la tradición aristotélica de influencia agustiniana, argumentando que quizás porque el Estagirita decía que la ética no era un saber propio para la juventud esta no se estudió, dejando con esa interpretación una dicotomía entre la tradición musulmana con la cristiana judaica y la del humanismo italiano, sin comprender la asimilación intercultural del aristotelismo ibérico de los siglos XII-XVI. Pagden interpreta el aristotelismo ibérico desde la academia y tiene aportaciones relevantes, pero es de notar que, en el ámbito de la aristocracia, autores como el marqués de Santillana -Iñigo López de Mendoza, 1398-1454-, padre de Diego Hurtado de Mendoza (1503-1575), leían latín, griego, hebreo y árabe. No olvidemos la relevancia de la acaudalada familia Mendoza en Granada, poseedores y lectores de las obras de Aristóteles que posteriormente llegan a ser virreyes en los principados americanos en el siglo XVI. También debe recordarse el interés de la familia Colón; el hijo de Cristóbal Colón, Fernando (1509-1539), formó la Biblioteca Colombina, de 1500 volúmenes, que contenía obras de Aristóteles. La Biblioteca del Escorial documenta obras de Aristóteles desde el siglo XIV, pero hubo un enciclopedismo ibérico en el siglo XIII y hoy se conocen traducciones de Aristóteles desde el siglo XII.

Lo relevante para los antecedentes del aristotelismo americano es que hay un protagonismo previo de obras de Aristóteles en las facultades de Artes y Derecho; el aristotelismo se dio en la corte de Aragón, que propiciaba la lengua griega; después, 
las enseñanzas bizantinas ya usaban la edición de Leonardo Bruni (1374-1444) y ya Nápoles y Sicilia habían impactado Hispania antes del siglo XVI con la lectura de obras del Estagirita. Así mismo, los textos del traductor renacentista bizantino, Ioannes Argyropolous, eran frecuentes. Es de notar que esto se dio en las facultades mencionadas. Otra cuestión es el rastreo de quiénes eran profesores en la facultad de Artes del siglo XV en Salamanca. La mayoría eran agustinos y, si de Salamanca sólo se revisa la facultad de teología para conocer a sus catedráticos, se yerra, ya que los profesores de teología eran fundamentalmente dominicos y tenían una impronta tomista mayor, pero esto no era lo que ocurría en las facultades de Artes y Derecho.

Como conclusión a esta aproximación de contexto, puede decirse que estudios posteriores a Pagden amplían la incidencia del aristotelismo ibérico; que éste cuenta con transversalidad de tradiciones; que América viene de una tradición aristotélica de corte aragonés que bebió del aristotelismo bizantino por dos vías fundamentales: la de Aviñón, Nápoles y Sicilia, con la tradicional tomista y la aristotélica previa que hemos mencionado, y que la orden agustina y a veces la franciscana desarrollaron en la facultad de Artes de la Universidad de Salamanca en el siglo XV.

El lector podría argumentar que el primer filósofo en impartir catedra prima en México ya con la Real Cédula de fundación de la Universidad-Alonso de la Veracruz-estudió en Salamanca en el siglo XVI cuando ya estaba el currículo con el impacto aristotélicotomista de Francisco de Vitoria, lo que en efecto se dio, pero hay algunos puntos que merecen señalarse al respecto: primero, que Alonso es agustino; que impartió clases en la facultad de Artes de la Universidad de Salamanca -no en la de teología- y que las obras sobre Aristóteles que realizó siguen la edición de Ioannes Argyropolous, traductor renacentista bizantino; que sus textos no siguen a Tomás de Aquino pese a que estructura sus obras de acuerdo al modelo tradicional medieval de Salamanca, por lo que vemos que tiene una posición electiva de Aristóteles en la cátedra; además, sus escritos no hacen un comentario ad literam del texto aristotélico como lo hacía Tomás, sino que realiza un seguimiento libre del texto, es decir, toma la fuente original para distintos fines e interpretación.

\section{l.1. Otras tradiciones aristotélicas llegadas a América}

Como la Real y Pontificia Universidad de México se funda a mediados del siglo XVI, sus académicos egresados de la Universidad de Salamanca, y los de Alcalá, la otra universidad española abierta al humanismo, ya cuentan con el conocimiento de otras tradiciones aristotélicas: el peripatetismo renacentista, el humanismo, las nuevas traducciones del griego y la publicación - gracias a la imprenta- de viejas interpretaciones aristotélico-latinas de corte neoplatónico (que fueron traídas a América y que se encuentran resguardadas en el Fondo Reservado de la Biblioteca Nacional de México, en el Centro de Estudios de Historia de México y en muchas Bibliotecas de la República esperando ser traducidas y trabajadas). Tenemos ya estudios serios que comprueban el protagonismo de las argumentaciones aristotélicas desde el siglo XVI. Merece especial atención el texto de Silvio Zavala La filosofía política de la conquista (1977) y el libro de A. Pagden La caída del hombre natural. El indio americano y los origenes de la etnología comparativa (1997), que prueban que la organización colonial siguió los criterios de la Política de Aristóteles, que el corpus del Estagirita fue el paradigma filosófico a partir del cual pensaron la realidad descubierta. Pero los textos europeos contemporáneos que documentaron los distintos aristotelismos, por ejemplo el de Schmitt, Aristotle and the Renaissance (Cfr. Schmitt, 1983; 1971), que abarcan el aristotelismo del siglo XIV al XVII, omiten cualquier mención de un aristotelismo en América. Schmitt mismo da las categorías específicas del aristotelismo renacentista: se trata de un aristotelismo que no es secuencia del medieval; un aristotelismo que se adapta a los principios del Renacimiento; un aristotelismo con un desarrollo interno y original, separado de influencias externas; un aristotelismo con diferencia en métodos 
4. Sobre este punto Edmundo O'Gorman dice que Las Casas da el argumento global de todo el tratado. Cfr. también Hernández Toledo (1967) y (2002), y De Sepúlveda $(1996: 23,70)$. En realidad toda la argumentación sepulvediense del tratado se ampara en Aristóteles (cfr. Pol., l-1). Finalmente, cfr. De Sigüenza y Góngora (1984: puntos 13 y 111-113).

5. Una selección y clasificación de argumentos aristotélicos en la Nueva España se encuentra desarrollada en Aspe Armella (2018: 397-404). y actitudes respecto del corpus. Schmitt concede que se trata de un aristotelismo que fue internacional: italiano, inglés, salmantino; católico, protestante; en la corte, en la cátedra, en las discusiones y polémicas, en la producción literaria y en las mentalidades. No obstante, nada dice del aristotelismo en América (Cfr. Schmitt, 1971: 5-7, 87).

La necesidad de rastrear el aristotelismo en América no responde a un deseo de reposicionar al continente (excluido, por cierto, por los ilustrados europeos hasta llegar a Hegel), sino a la necesidad filosófica de comprender y aprender las aproximaciones que se han dado de argumentos aristotélicos para penetrar más en la realidad de la que se ocupan. Es decir, ver si aportan algo, si tienen vigencia, y saber cuáles han sido las reflexiones filosóficas de nuestra tradición académica.

Lo que es un hecho, porque está documentado (Cfr. Plaza y Jaén, 1931), es que el currículo de las facultades de la Universidad de México incluía transversalmente el estudio de las obras de Aristóteles y que, en la facultad de Artes y Teología, era obligatorio el corpus aristotelicum del que se hacían siempre comentarios. Esto viene documentado también por los estudios de José María Gallegos Rocafull y Mauricio Beuchot, por lo que debe concluirse que Aristóteles fue el protagonista fundamental del currículo de la Universidad de México (Cfr. Gallegos Rocafull, 1974; Beuchot, 1996).

Además del currículo universitario, son famosas las polémicas habidas en el período novohispano de México: la disputa entre Juan Ginés de Sepúlveda y Bartolomé de las Casas, por ejemplo, que tiene como telón de fondo dos interpretaciones de Aristóteles en ocasión de la invasión imperial, la Conquista y la racionalidad del indio americano. Por mencionar otras dos de los siglos coloniales, cabe señalar, primero, la Libra Astronómica y Filosófica de Carlos de Sigüenza y Góngora que, en el siglo XVII, refuta a científicos europeos con argumentos aristotélicos; por último, hay una tradición de obras naturalistas que siguen a Aristóteles y que abarcan desde el siglo XVI con Bernardino de Sahagún y Francisco Hernández hasta el XVIII con Francisco Javier Clavijero. Me interesa señalar esta tradición y profundizaré en ella en el siguiente apartado; por ahora podemos decir que comienza con la Historia general de las cosas de la Nueva España de Bernardino de Sahagún, con Bartolomé de Las Casas en su Apologética historia sumaria y, en especial, con la obra de Francisco Hernández (15401600): Historia Natural y Moral de las Indias: en que se tratan las cosas notables del cielo y elementos, metales, plantas y animales dellas, y los ritos y ceremonias, leyes y gobierno, $y$ guerras de los indios (De las Casas, 1974: I-1). ${ }^{4}$

\section{Algunas argumentaciones internas que muestran la pertinencia de revisar el aristotelismo novohispano}

Pasemos ahora a las cuestiones internas del tema que nos ocupa. ${ }^{5}$ Anthony Pagden demostró ya la existencia de argumentaciones aristotélicas asépticas en la disputa entre Bartolomé de las Casas y Juan Ginés de Sepúlveda (Cfr. Pagden, 1997). Más allá de los argumentos lascasianos sobre el hombre universalmente creado imago Dei basado en el Génesis y de su impronta agustiniana en la lucha evangelizadora en América, inspirándose en la Ciudad de Dios de san Agustín, Las Casas lleva la disputa sobre la racionalidad del indio al tema del esclavo por naturaleza de Politica I superando el criterio español de la insuficiencia psicológica indígena por una falta del thymós aristotélico. Una vez que Las Casas prueba que los indios tienen leyes, artes, magistrados, escritura y técnica, condiciones del ciudadano aristotélico, acude a la cuestión del thymós aristotélico señalando que el Estagirita argumentaba que por los climas tórridos se carecía de la fuerza vital para autogobernarse y que los faltos de espíritu libertario (en la medida en que tienen un intelecto inferior) desarrollan cuerpos más robustos para dedicarse a las tareas manuales. 
Siguiendo ese criterio de Aristóteles, Las Casas describe una anatomía indiana frágil y poco corpulenta como era el criterio anatómico aristotélico del hombre civilizado; prueba que en el indio americano la fuerza y corpulencia no se desarrollaron porque tenían plena racionalidad.

En la polémica sobre la Conquista se ataba el thymós a un discurso que daba al hombre occidental europeo mayor consciencia para realizar los fines de la vida humana. Las Casas retoma la interpretación de De Anima II 412a20-30 con el pasaje de la Política 1454a5 en el que se dice que la vida es acción y no producción, y que el esclavo por ende es un subordinado para la acción. Pero distingue entre esclavos por convención y esclavos por naturaleza, llevando así la discusión al tema del bárbaro, pues considera que algunos parecen ser esclavos por naturaleza, pero no lo son. Así, el bárbaro o extranjero aristotélico le es más propio para dirimir el tema de la esclavitud y da cuatro sentidos de bárbaro: aquéllos que lo parecen por la diversidad de su lengua y costumbres, pero que no lo son (es decir, sólo están privados de la forma de vida occidental); aquéllos privados de lenguaje y escritura; aquéllos privados de la fe, y aquéllos que por naturaleza no tienen autodominio ni razón plena. Estos últimos, muy raros y escasos en principio, considera que deben esclavizarse para evitar que se hagan daño a sí mismos y a otros.

Por último, Las Casas argumenta, basado en ENVIII-II 1161b1-10 que, aunque Aristóteles no acepta amistad entre el amo y el esclavo, sí dice que puede haberla entre el amo y el esclavo en cuanto hombres. La argumentación la completa en Apologética historia sumaria, donde describe la excelencia indiana y su capacidad de deliberación relativa a los fines propios de la buena vida aristotélica. Esa obra es la prueba de facto de que, a la luz de la Política, el indio americano reúne las condiciones del ciudadano. Las Casas da allí razones anatómicas de proporción para mostrar su capacidad de vida virtuosa (De las Casas, 1997). ${ }^{6}$

Pasemos ahora a la clasificación naturalista que hace Francisco Hernández Toledo (ca. 1514-1587), médico, botánico y ornitólogo que estudió medicina en la Universidad de Alcalá. Nombrado "protomédico de las Indias, islas y tierra firme del mar océano" por Felipe II, vino a Nueva España en 1570. Su obra más reconocida fue Compendio de Historia Naturaly Moral de las Indias (Cfr. Hernández Toledo, 1967 y 2002). De acuerdo con algunos especialistas, Hernández es precursor de la botánica y la materia médica moderna (Cfr. López Piñeiro y Pardo Tomás, 1996). La relevancia de este autor -que sigue a Aristóteles para la clasificación de los minerales, plantas y animales de América- está en que asume la tradición naturalista previa de Bernardino de Sahagún y analiza las propiedades naturales de las cosas de América registrando los nombres de éstas en la herbolaria y medicina náhuatl, preservando así la lengua y aportaciones científicas de los pueblos originarios. A pesar de que se le ha denominado "aristotélico", su obra tiene una finalidad práctica que busca "la salud de los pueblos de América". Conformado por cuatro libros, este texto se apoya en los treinta y siete libros de la Historia Natural de Plinio, a quien Hernández tradujo en Nueva España durante su estancia de tres años. Además, publicó otra obra, Compendio de filosofía moral según Aristóteles. ${ }^{7} \mathrm{Y}$ también escribió Cuestiones de los estoicos, Problemas de los estoicos y Problemas o erotemas filosóficos según la mente de los peripatéticos y los principios de Aristóteles (Cfr. Hernández Toledo, 1967). Sus obras prueban un aristotelismo de cuño renacentista que tiene la intención de "recuperar al verdadero Aristóteles", apartarse de una filosofía que "sólo se basa en el Evangelio" y que tiene notas sobre los textos aristotélicos (de los que Elsa Cecilia Frost (1984) afirma que son meras anotaciones y apuntes de clase).

En esta obra, los temas que trata son el bien, los fines, la felicidad, las virtudes, los vicios y los actos voluntarios e involuntarios; en mi opinión, pese al cuño aristotélico
6. Para el tema del thymós, cfr. Mauri (2016), Dobbs (1994) y Frank (2004).

7. La obra ha sido trabajada por diversos filósofos. Cfr. Esquivel Estrada (2006). 
que pretende, y a pesar de poner el fin último del hombre en la vida política, termina por argumentar con las razones probables de la bienaventuranza en Dios. Empero, autores especializados han subrayado el seguimiento aristotélico puntual de sus clasificaciones naturales (Cfr. Durand-Forest y Durand, 1993).

Llama la atención el método utilizado en la Historia Natural. En los cuatro libros se analiza la materia medicinal de hierbas aromáticas (libro I), las hierbas medicinales con sabor agudo (libro II), las hierbas que tienen sabores salado y dulce (libro III), y el último libro, dividido en dos partes, trata de 1) animales y las partes de estos que sirven para la medicina, y 2) los minerales. Con rigor, pone después una tabla de todos los medicamentos simples que descubre en la tradición indígena; posteriormente presenta una lista, en orden alfabético, y una tabla con todo género de enfermedades y cómo curarlas (por ejemplo, cómo bajar la temperatura y la fiebre). Por último, incluye unas páginas con erratas, poniendo el término náhuatl que escribió y a un lado como debe escribirse este (por ejemplo, en la p. 1 línea 7 dice Coapantliy debe decir Coapatli). Para un autor del siglo XVI, es sorprendente el rigor clasificatorio y de método en la investigación, pues sólo estuvo tres años en Nueva España (y con enormes problemas de financiamiento a causa de los celos de las autoridades políticas por haber sido enviado por el rey).

La aportación de este autor, cara al aristotelismo que rastreo, es su observación, sus anotaciones y la ulterior clasificación de las plantas de América. Es notable cómo realiza una investigación propia de los médicos árabes que recuperaron el aristotelismo naturalista y la manera de seguir a autores naturalistas latinos como Plinio. Esta tradición aristotélica de Nueva España habría de analizarse con más profundidad, ya que instaura las observaciones sobre la herbolaria indígena con metodología clasificatoria de Aristóteles. Desgraciadamente, la documentación e ilustración de las plantas realizadas al modo europeo por un tlacuilo náhuatl dieron con que se apreciaran más por los dibujos, quedando mudo el valor del método y la significación científica; el italiano Nardi publicó las ilustraciones a colores en Italia y esto es lo que perduró.

Termino señalando dos argumentaciones relevantes de Alonso de la Veracruz: en sus Investigaciones acerca del alma, Alonso (autor que como venimos diciendo las hace siguiendo el texto de Aristóteles que tradujo del griego al latín el bizantino Argyropolus) hace una aportación relevante cuando abre diciendo que el estudio del alma humana debe ubicarse en la filosofía natural, ya que el hombre es un compuesto de materia y forma, por lo que no puede separarse su estudio de la materia (Cfr. De la Veracruz, 1976). La otra aportación destacable del agustino está relacionada con su obra Recognitio summularum I-1, de 1554, en la que rechaza que la lógica sea una ciencia y reitera que sólo es un arte y que la tradición medieval ha errado en esa interpretación alejada de Aristóteles (Cfr. De la Veracruz, 1942). ${ }^{8}$ En la primera relectio de América, que en su tiempo consistía en una conferencia inaugural del curso de la facultad de Teología y Artes de la Real y Pontificia Universidad de México (en este caso del primer curso de 1553-1554), Alonso presenta una disertación que posteriormente ampliara para su publicación bajo el título de De dominio infidelium et iusto bello, o Sobre el dominio de los infieles y guerra justa (cfr. De la Veracruz, 2007). Alonso echará mando del axioma aristotélico de Pol., I-3 que pone el fin de la vida política en el bien común de la polis estableciendo criterios republicanos (cfr. De dominio, puntos 1-7 y 13-16). También en sus primeros curso de la facultad de Artes en 1554, publicados bajo el nombre de Recognitio summularum (de 1554) y en Dialectica resolutio (1554), del segundo semestre, propone una lógica simplificada separada de las complejas y abstractas especulaciones de la lógica escolástica. Aristóteles sirve a Alonso para depurar muchos de los vicios en que había caído la Escolástica, y su selección del traductor renacentista Argyropolous es signo del afán renovador humanista que inspiró el aristotelismo fundacional americano. 
Como conclusión a esta segunda parte de mi escrito puede decirse que hay una tradición aristotélica en México desde los inicios de la Real y Pontificia Universidad de México, que, si bien se distingue de las otras tradiciones occidentales y orientales, ha bebido de ellas haciendo una peculiar formulación de cara al nuevo orbe observado y al tema del otro americano al que se enfrentaban los pensadores, viajeros, académicos y políticos de la administración colonial. Aristóteles sirvió como andamiaje tanto para la elaboración del plan político colonial como para la clasificación del orbe observado y del conocimiento de las funciones de los seres naturales y sus habitantes. Esta tradición se encuentra esperando ser traducida y comentada en la serie de documentos inéditos que están en los fondos reservados de los archivos nacionales. 


\section{Bibliografía}

》 Aristóteles. (1985). Ética nicomáquea. Madrid: Gredos.

" Aristóteles. (1988). Política. Madrid: Gredos.

» Aristóteles. (2014). Acerca del alma. Madrid: Gredos.

»Aspe Armella, V. (2014). El aristotelismo de la primera etapa de la Universidad de Salamanca. En V. Aspe Armella, \& M. I. Zorroza, Francisco de Vitoria en la Escuela de Salamanca y su proyección en Nueva España (pp 47 - 60). Pamplona: EUNSA.

» Aspe Armella, V. (2018). Aristóteles y Nueva España. San Luis Potosí: UASLP.

»Beuchot, M. (1996). Historia de la filosofía en el México colonial. Barcelona: Herder.

"Cabanchik, S. M. (2015). La filosofía en lengua española (o castellana). Avatares filosóficos, 2, 14-22.

"Candia Baeza, C. (2007). Filosofía, identidad y pensamiento político en Latinoamérica. Polis, 18.

" Castillo Vegas, J. L. (2005). Aristotelismo político en la Universidad de Salamanca del siglo XV: Alfonso de Madrigal y Fernando de Roa. La corónica: A Journal of Medieval Hispanic Languages, Literatures, and Cultures, 39-52.

»Coll, J. M. (1944). Escuelas de lenguas orientales en los siglos XIII y XIV. Período postraymundiano. Analecta sacra tarraconensia: Revista de ciències historicoeclesiàstiques, 77-98.

» De la Veracruz, A. (1942). Investigación filosófico-natural. Los libros del alma. Libros I $y$ Il. Ciudad de México: UNAM.

»De la Veracruz, A. (1976). Investigaciones acerca del alma. (O. Robles, Ed.) Ciudad de México: UNAM.

»De la Veracruz, A. (2007). De dominio infidelium et iusto bello. (R. Heredia Correa, Ed., \& R. Heredia Correa, Trad.) Ciudad de México: UNAM.

"De la Veracruz, A. (2007). Dialectica resolutio. Ciudad de México: UNAM.

»De las Casas, B. (1974). Apologética historia sumaria. Ciudad de México: UNAM.

"De las Casas, B. (1997). Tratados filosóficos. Ciudad de México: FCE.

»De Sepúlveda, G. (1996). Tratado sobre las justas causas de la guerra contra los indios. Ciudad de México: FCE.

» De Sigüenza y Góngora, C. (1984). Libra astronómica y filosófica. Ciudad de México: UNAM.

"Dobbs, D. (1994). Natural Right and the Problem of Aristotle's Defense of Slavery. The Journal of Politics, 1 (6), 69-94.

» Durand-Forest, J. d., \& Durand, E. J. (1993). Aperçu de l'Histoire Naturelle de la Nouvelle-Espagne d'après Hernández, les informateurs indigènes de Sahagún et les auteurs du Codex Badianus. Avec Illustrations. En M.-C. Benassy, \& A. Saint-Lu, Nouveau Monde et renouveau de l'histoire naturelle (pp 3-28). París: Service des Publications de la Sorbonne Nouvelle Paris III.

»Esquivel Estrada, N. H. (2006). Francisco Hernández (1518-1587) y su filosofía moral (comentarios al primer libro de su obra Compendio de philosophia moral 
según Aristóteles en las ethicas que escribió a Nicómaco y su relación con la Ética nicomaquea de Aristóteles). Pensamiento novohispano, 83-94.

»Frank, J. (2004). Citizens, Slaves and Foreigners. Aristotle on Human Nature. American Political Science Review, 1 (8), 91-104.

»Frost, E. C. (1984). Los intereses filosóficos de Francisco Hernández. En F. Hernández Toledo, Obras completas (Vol. VI). Ciudad de México: UNAM.

"Gallegos Rocafull, J. M. (1974). El pensamiento mexicano en los siglos XVI y XVII. Ciudad de México: UNAM.

"Gaos, J. (1996). Obras completas VIII. Filosofía mexicana de nuestros días. En torno a la filosofía mexicana. Sobre la filosofía y la cultura en México (Vol. VIII). Ciudad de México: UNAM.

» García Ballester, L. (1994). A marginal learned medical world: Jewish, Muslim and Christian medical practitioners, and the use of Arabic medical sources in late medieval Spain. En L. García Ballester, R. French, J. Arrizabalga, \& A. Cunningham, Practical Medicine from Salerno to the Black Death (pp 353-394). Nueva York: Cambridge University Press.

" Gázquez, M. (1995). Traducciones árabo-latinas en Murcia. Filología mediolatina. Rivista della Fondazione Ezio Franceschini, 2, 249-258.

"Hernández Toledo, F. (1967). Obras completas. Ciudad de México: UNAM.

"Hernández Toledo, F. (2002). The Mexican Treasury: The Writings of Dr. Francisco Hernández. (S. Varey, Ed., S. Varey, R. Chabrás, \& C. Chamberlin, Trads.) Stanford: Stanford University Press.

» Herrero Prado, J. L. (1998). Pero Díaz de Toledo, señor de Olmedilla . Revista de literatura medieval, $X, 101-115$.

» López Piñero, J. M., \& Pardo Tomás, J. (1996). La influencia de Francisco Hernández (1515-1587) en la constitución de la botánica y la materia médica modernas. Valencia: Instituto de Estudios Documentales e Históricos sobre la Ciencia. Universitat de València-CSIC.

» Mauri, M. (2016). La esclavitud natural. Una revisión de las tesis de Aristóteles. Ideas y valores, 162 (65), 161-187.

» Padgen, A. R. (1975). The Diffusion of Aristotle's Moral Philosophy in Spain, ca. 1400 - ca. 1600. Traditio, 31, 287-313.

"Pagden, A. R. (1997). La caída del hombre natural. El indio americano y los orígenes de la etnología comparativa. Madrid: Alianza.

» Plaza y Jaén, C. B. (1932). Crónica de la Real y Pontificia Universidad de México. Ciudad de México: UNAM.

" Round, N. G. (1966). Pero Díaz de Toledo: A Study of the Fifteenth-Century Converso Translator in his Background. Oxford: Tesis doctoral no publicada.

» Sabido, C. (2016). El pensamiento ético-político de Alfonso de Madrigal. Pamplona: EUNSA.

» Schmitt, C. B. (1971). A Critical Survey and Bibliography of Studies on Renaissance Aristotelianism, 1958 - 1969. Padua: Antenore.

» Schmitt, C. B. (1983). Aristotle and the Renaissance. Cambridge: Harvard Universit Press.

»Zavala, S. (1977). La filosofía política de la conquista. Ciudad de México: FCE. 
